\title{
Midterm results of transapical aortic valve replacement via real-time magnetic resonance imaging guidance
}

\author{
Keith A. Horvath, MD, ${ }^{\mathrm{a}}$ Dumitru Mazilu, PhD, ${ }^{\mathrm{b}}$ Michael Guttman, MS, ${ }^{\mathrm{c}}$ Arthur Zetts, ${ }^{\mathrm{d}}$ Timothy Hunt, ${ }^{\mathrm{d}}$ and \\ Ming $\mathrm{Li}, \mathrm{PhD}^{\mathrm{b}}$
}

Objective: Percutaneous valve replacements are presently being evaluated in clinical trials. As delivery of the valve is catheter based, the safety and efficacy of these procedures may be influenced by the imaging used. To assist the surgeon and improve the success of the operation, we have performed transapical aortic valve replacements using real-time magnetic resonance imaging guidance.

\begin{abstract}
Methods: Twenty-eight swine underwent aortic valve replacement by real-time magnetic resonance imaging on the beating heart. Stentless bioprostheses mounted on balloon-expandable stents were used. Magnetic resonance imaging $(1.5 \mathrm{~T})$ was used to identify the critical anatomic landmarks. In addition to anatomic confirmation of adequate placement of the prosthesis, functional assessment of the valve and left ventricle and perfusion were also obtained with magnetic resonance imaging. A series of short-term feasibility experiments were conducted $(n=18)$ in which the animals were humanely killed after valve placement and assessment by magnetic resonance imaging. Ten additional animals were allowed to survive and had follow-up magnetic resonance imaging scans and confirmatory echocardiography at 1,3, and 6 months postoperatively.
\end{abstract}

Results: Real-time magnetic resonance imaging provided superior visualization of the landmarks needed. The time to implantation after apical access was $74 \pm 18$ seconds. Perfusion scanning demonstrated adequate coronary flow and functional imaging documented preservation of ventricular contractility in all animals after successful deployment. Phase contrast imaging revealed minimal intravalvular or paravalvular leaks. Longer term results demonstrated stability of the implants with preservation of myocardial perfusion and function over time.

Conclusions: Real-time magnetic resonance imaging provides excellent visualization for intraoperative guidance of aortic valve replacement on the beating heart. Additionally, it allows assessment of tissue perfusion and organ function that is not obtainable by conventional imaging alone. (J Thorac Cardiovasc Surg 2010;139:424-30)

Valve replacement in the beating heart using either a percutaneous or left ventricular apical access has been reported. ${ }^{1-9}$ These minimally invasive approaches reduce trauma and speed recovery for the patient. Compared with the percutaneous transfemoral approach, transapical aortic valve replacement provides a direct and short access to the native valve. It requires general anesthesia, but this approach may be more applicable to a wider range of patients because of the lack of physical anatomic limitations. In addition, the

From the National Heart, Lung, and Blood Institute, National Institutes of Health: the Cardiothoracic Surgery Research Program ${ }^{\mathrm{a}}$; the Biomechanical Section, ${ }^{\mathrm{b}}$ Cardiothoracic Surgery Research Program; the Laboratory of Cardiac Energetics ${ }^{\text {; }}$; and the Laboratory of Animal Medicine and Surgery, ${ }^{\mathrm{d}}$ Bethesda, Md.

Disclosures: None.

Supported through the Intramural Research Program of the National Heart, Lung, and Blood Institute, National Institutes of Health, Department of Health and Human Services.

Read at the Thirty-fifth Annual Meeting of The Western Thoracic Surgical Association, Banff, Alberta, Canada, June 24-27, 2009.

Received for publication June 19, 2009; revisions received July 27, 2009; accepted for publication Aug 9, 2009; available ahead of print Dec 7, 2009.

Address for reprints: Keith A. Horvath, MD, Director, Cardiothoracic Surgery Research Program, National Heart, Lung, and Blood Institute, National Institutes of Health, 10 Center Dr-MSC 1454, Building 10 CRC, Room 2 N-246, Bethesda, MD 20892 (E-mail: khorvath@nih.gov).

$0022-5223 / \$ 36.00$

Copyright (c) 2010 by The American Association for Thoracic Surgery doi:10.1016/j.jtcvs.2009.08.005 transapical approach permits the potential implantation of a conventional prosthesis that has a known durability and proven success rate.

Typically, the imaging used for percutaneous valve placement is fluoroscopy, which provides 2-dimensional visualization with little soft tissue contrast. Contrast agents must be repeatedly injected to determine the location of the aortic annulus and coronary ostia. The patient and physician are exposed to radiation during the intervention. Rapid ventricular pacing to unload the ventricle, which is considered fundamental for the success of the procedure, has precipitated cardiac arrest in some high-risk patients. Moreover, additional imaging and expertise with echocardiography is required for postintervention valvular assessment.

Magnetic resonance imaging (MRI) provides better anatomic detail than fluoroscopic or echocardiographic imaging without additional risk of radiation and contrast reaction. As a result of the clarity of MRI, image interpretation is easy for anyone familiar with the surgical anatomy. The development of real-time MRI allows this imaging modality to provide intraoperative guidance for delivery of prosthetic aortic valves. ${ }^{10,11}$ MRI also provides the ability to assess results, such as ventricular and valvular function and myocardial perfusion immediately after intervention. To demonstrate 


\section{Abbreviations and Acronyms \\ Gd-DTPA = gadolinium with diethylenetriamine penta-acetic acid \\ MR = magnetic resonance \\ MRI = magnetic resonance imaging}

how real-time MRI can assist the surgeon and improve the success of the operation, we have performed transapical aortic valve replacement using real-time MRI guidance. We report our real-time MRI-guided transapical aortic valve experiment technique and its midterm results.

\section{MATERIALS AND METHODS}

\section{Imaging System, Prosthetic Valve, and Devices}

A 1.5 T Magnetom Espree MRI (Siemens Medical Solutions, Munich, Germany) was used for the intervention. This magnet design, with a short $(120 \mathrm{~cm})$ and wide $(70 \mathrm{~cm})$ bore, makes surgical access to the patient within the magnet feasible. High-quality images can be obtained at 5 to 10 frames per second with low latency. In addition to providing standard MR sequences, a fully interactive, real-time MRI system connected to the scanner provides a real-time interactive imaging sequencing. ${ }^{12,13}$ This system comprises an interactive user interface, an operating room large-screen display, gated pulse sequences, and image reconstruction software. Multiple oblique slices can be obtained in rapid succession and can be simultaneously displayed in a 3-dimensional rendering to provide optimal 3-dimensional ana- tomic information. Image contrast, image plane orientations, acquisition speed, 3-dimensional rendering, and device tracking can be readily adjusted as needed during scanning. Standard MR sequences are used for preoperative surgical planning and postplacement evaluation; in between this standard scanning, real-time MRI provides feedback on the progress of the procedure.

Since the intervention is performed in the MRI scanner, the instruments and materials must be compatible with the magnetic field. Commercially available 21- to 25-mm stentless bioprostheses (Toronto SPV; St Jude Medical, Inc, Minneapolis, Minn; or Freestyle aortic root bioprosthesis; Medtronic, Inc, Minneapolis, Minn) were selected on the basis of the aortic valve size scanned with MRI. These bioprosthetic valves were mounted on an analogous-sized, platinum-iridium stent (Cheatham Platinum; NuMed, Hopkinton, NY). Small austenitic stainless steel fragments $(0.2 \mathrm{~mm})$ were welded on the side of the stent between the bioprosthetic valve commissures. This paramagnetic marker is visible as a dark signal in the MRI and is used to indicate the orientation of the valve/stent. The bioprosthesis and stent were then circumferentially compressed over 2-stage balloon-tipped catheters (NuMed, 25-30 mm outer diameter, $50 \mathrm{~mm}$ long). Figure 1 shows a Medtronic valve mounted on a NuMed platinum-iridium stent and its MR image. A central guidewire allowed tracking of the balloon-tipped catheter antegradely across the native aortic valve.

The delivery device consists of a straight plastic rod, outside of which is a sheath protecting the bioprosthetic valve before it is deployed. The length of the delivery device is $60 \mathrm{~cm}$. The diameter of the delivery device is 9.5 $\mathrm{mm}$ and it fits into a $10-\mathrm{mm}$ trocar. The inner rod has a $6.35-\mathrm{mm}$ central channel for the guidewire-directed balloon-tipped catheter. At the distal end of the inner rod, a locking system is used to prevent relative motion between the catheter and the rod itself. The translation and rotation of the rod directly relate to the translation and rotation of the balloon catheter. The

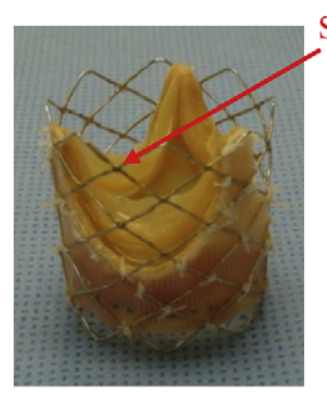

A

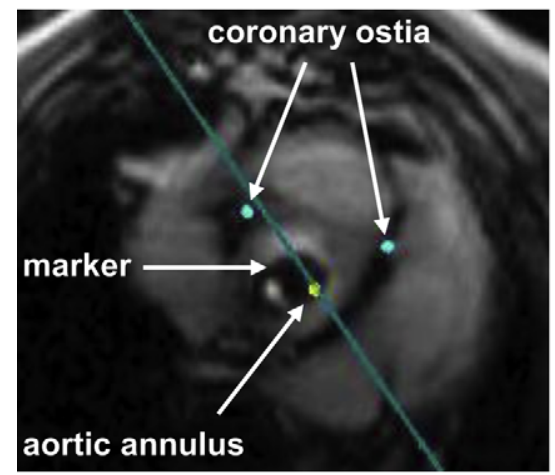

C
Stainless steel marker

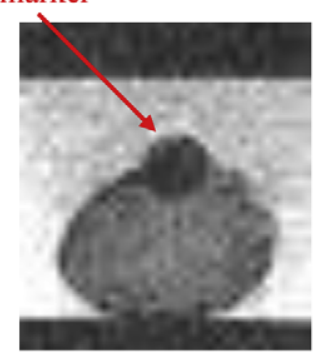

B

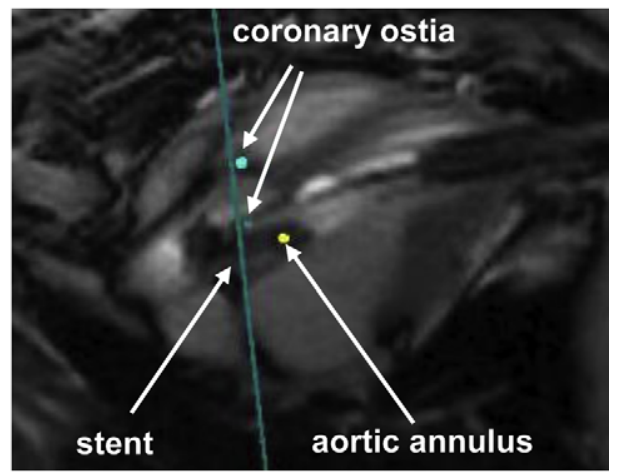

D

FIGURE 1. Bioprosthesis mounted on a platinum-iridium stent. A stainless steel marker welded on the side of the stent between the commissures (A). The marker is visible as a dark signal in the MRI and indicates the orientation of the prosthesis (B). The short-axis view and long-axis view of the prosthesis with a passive marker under real-time MRI are shown in parts $\mathrm{C}$ and D, respectively. In parts $\mathrm{C}$ and D, 2 blue dots are the digital markers that indicate the coronary ostia and the yellow dot is the digital marker of the aortic annulus location. 
inner rod and the sheath are relatively tight with each other; a small rubber gasket is used to prevent blood leakage from the central channel.

\section{Preoperative Preparation}

All experiments were performed under protocols approved by the National Institutes of Health Animal Care and Use committee. After induction, 28 domestic pigs were intubated and anesthetized. Standard MR sequences were performed to obtain the orientation of the heart, evaluate ventricular and valve function, and locate the native valve annulus and the origin of the coronary arteries. By use of standard titanium surgical instruments via a 6-cm subxiphoid incision, the pericardium was opened and the apex of the heart was exposed. Two concentric purse-string sutures were placed around the apex, through which a 10-mm trocar (Ethicon Surgical Inc, Somerville, NJ) was inserted into the left ventricle. Typical time to complete this part of the procedure was 15 to 20 minutes.

Pre-scanning also allows setting up scan planes to be used for real-time imaging during valve implantation and follow-up myocardial perfusion and aortic flow imaging. Three imaging planes were prescribed for real-time imaging during implantation. Two of these planes were positioned to provide long-axis views of the left ventricle, showing the right coronary artery and left anterior descending coronary artery origins, respectively. The other plane provided an axial view of the aortic valve. The coronary ostia and aortic annulus location were digitally marked. These digital marks remained visible at all times in the 3-dimensional rendering and were used for anatomic reference.

\section{Valve Implantation}

The surgeon views the real-time imaging on a projection screen while manipulating the deployment device within the animal in the magnet. Three snapshots of the real-time MRI with multiple image planes are shown in Figure 2.

A guidewire is advanced through the trocar across the native aortic valve, after which the prosthetic valve and delivery system are advanced through the trocar. During implantation, the axial slice is shifted as needed to visualize the device and guide proper orientation of commissures with the help of the passive marker. The long-axis views are interactively modified to show the path of the delivery device, while keeping the coronary origins in view. The surgeon is in contact with the scanner operator by means of headphones and a microphone (Magnacoustics, Atlantic Beach, NY) to request changes in the imaging planes as needed.

Once in place, the balloon is partially inflated by using normal saline mixed 100:1 with an MR contrast agent, gadolinium with diethylenetriamine penta-acetic acid (Gd-DTPA; Magnavist, Berlex Inc, Montville, $\mathrm{NJ})$. The position is reconfirmed to be ideal, and the balloon is then fully expanded and the valve/stent deployed while monitoring with the realtime MRI. The balloon is then deflated. The catheter and guidewire are removed through the trocar. Ventricular function is immediately assessed with the real-time imaging.

During the procedure, the animals were monitored with an electrocardiogram, oxygen saturation, end-tidal carbon dioxide, systemic and left ventricular blood pressure, and arterial blood gas analysis.

\section{Postplacement Validation}

After placement of the valve, the trocar was removed and the apex closed with the purse-string sutures. Postplacement images were acquired to confirm the positions of the prostheses and the valvular and heart function. In addition to anatomic confirmation of adequate placement of the prosthetic valve in relation to the aortic annulus and the coronary arteries, functional assessment of the valve and left ventricle was also obtained with MRI. Gated cine-MRI was used to assess mitral valve function and myocardial function. Phase contrast cine-MRI was used to identify flow through the new valve as well as intravalvular or paravalvular regurgitation. An MR first-pass perfusion $\operatorname{scan}^{14}$ was performed during intravenous injection of

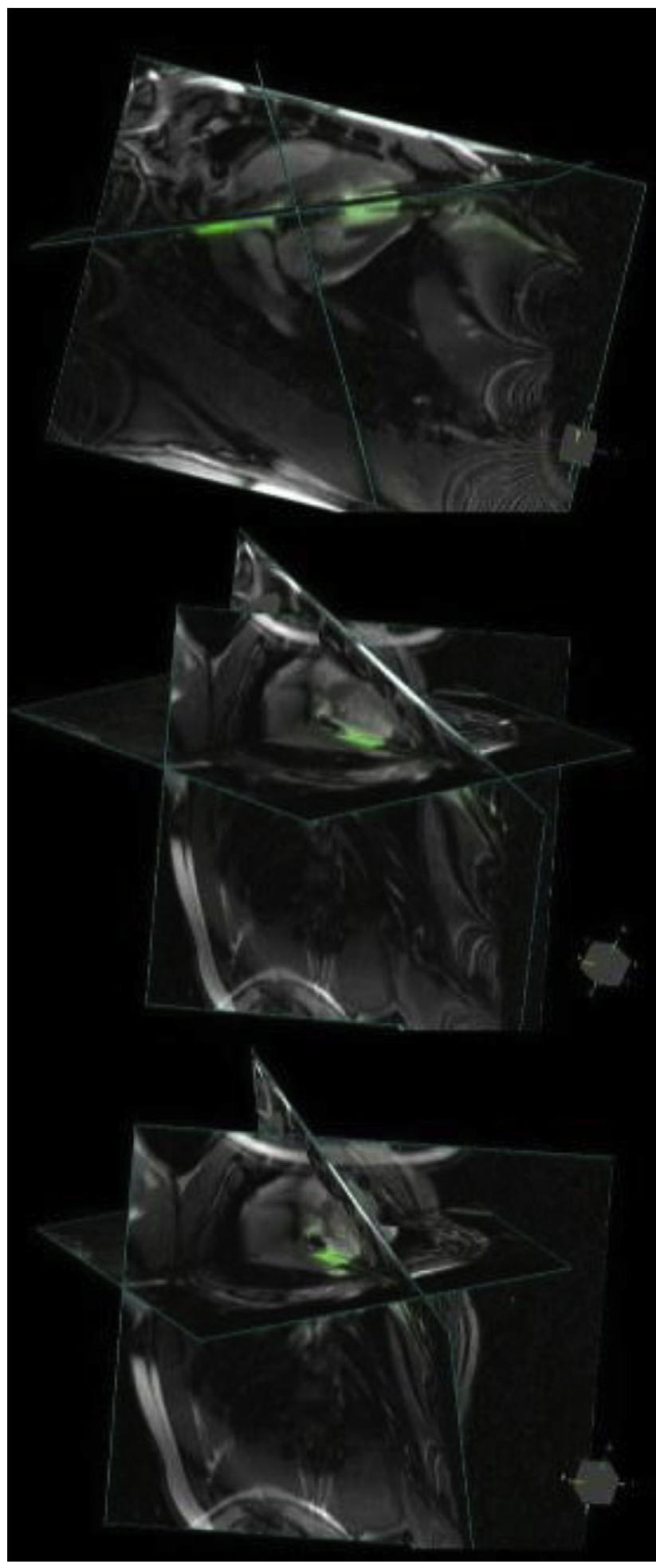

FIGURE 2. Real-time MRI provided superior visualization for implanting the aortic valve prosthesis. Snapshots show multiple image planes displayed at their relative 3-dimensional position. The 3-dimensional rendering provides 3-dimensional anatomic information. 


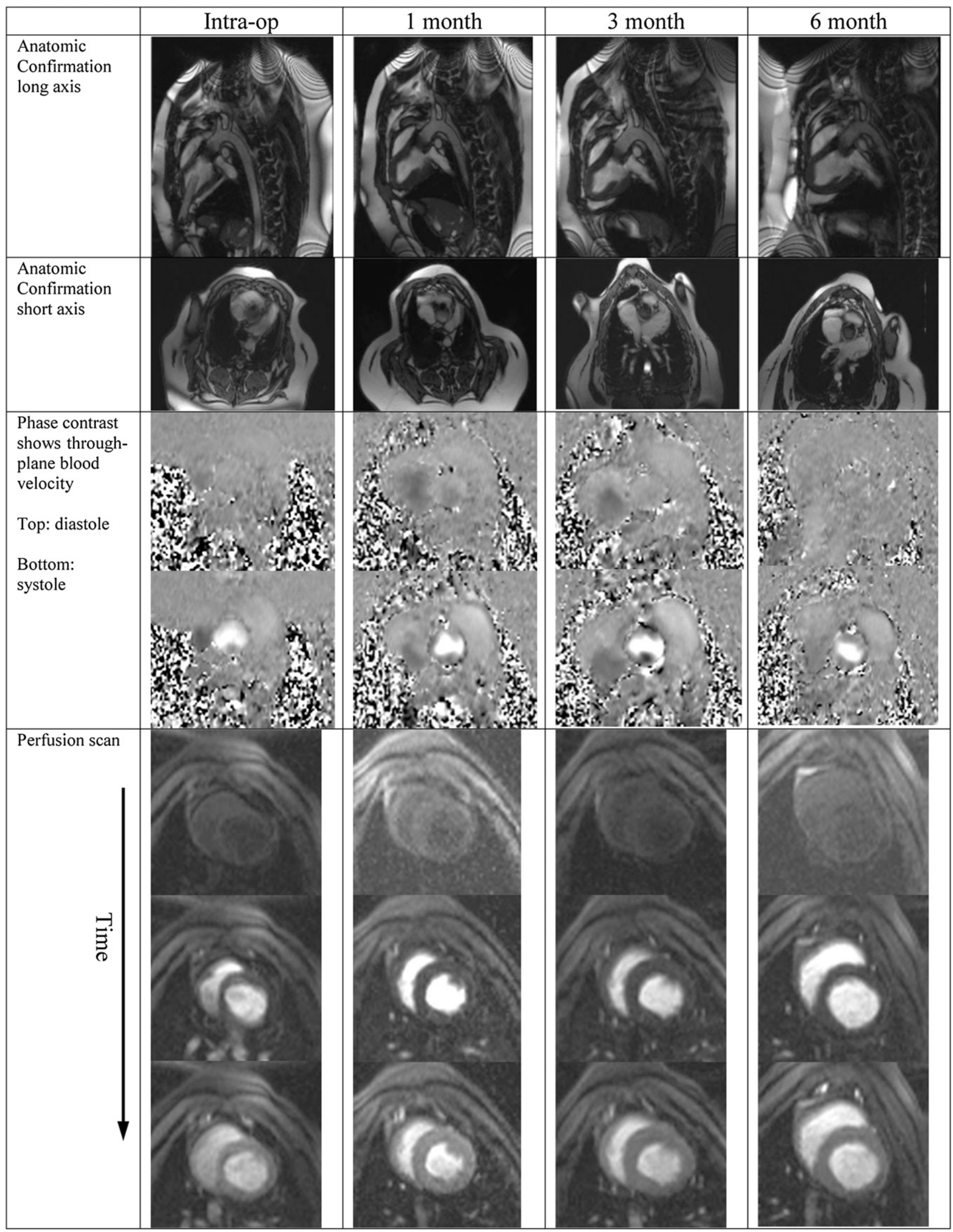

FIGURE 3. Long-term follow-up MRI scans at 1,3 , and 6 months postoperatively. 
TABLE 1. The ventricular and valvular results

\begin{tabular}{lccccc}
\hline & LVDD $(\mathbf{m m})$ & AV root $(\mathbf{m m})$ & EF $(\%)$ & AV peak gradient & AV mean gradient \\
\hline 1 mo & $43 \pm 10$ & $28 \pm 1.1$ & $63 \pm 4.1$ & $9.3 \pm 3.1$ & $4.4 \pm 1.5$ \\
3 mo & $45 \pm 9.9$ & $27 \pm 1.5$ & $64 \pm 2.2$ & $15.0 \pm 5.7$ & $7.3 \pm 3.1$ \\
6 mo & $47 \pm 9.9$ & $27 \pm 1.9$ & $64 \pm 3.8$ & $12.0 \pm 5.2$ & $5.6 \pm 2.6$ \\
ANOVA $P$ value & .817 & .688 & .912 & .133 & .144 \\
\hline
\end{tabular}

Measurements were based on magnetic resonance imaging and confirmatory echocardiographic data. $L V D D$, Left ventricular diastolic dimension; $A V$, aortic valve; $E F$, ejection fraction; ANOVA, analysis of variance.

Gd-DTPA contrast agent to confirm that myocardial blood flow was intact to all segments of the myocardium.

\section{Experimental Follow-up}

A series of short-term feasibility experiments was conducted $(n=18)$ in which the animals were humanely killed after valve placement and MRI assessment. Ten additional animals were allowed to survive for long-term follow-up. At 1 and 3 months postoperatively, follow-up MRI scans and transthoracic echocardiograms were acquired while at 6 months postoperatively MRI scans and confirmatory 2- and 3-dimensional transesophageal echocardiograms were acquired. Retrospectively, gated cine-MR, phasecontrast cine-MR, and MR first-pass perfusion scanning during intravenous injection of Gd-DTPA contrast agent were repeated at those time points to confirm the position of the prostheses and the valvular and heart function.

\section{RESULTS}

Real-time MRI provided better visualization of the landmarks needed to implant the aortic valve prostheses than fluoroscopy or echocardiography. The passive marker on the stent is a superb indicator of the valve orientation in MRI at the time of placement and can be used to determine whether the valve migrates over time. The time to implantation after the apical access was obtained to deployment of the valve was $74 \pm 18$ seconds. This includes the time to correctly orient the prosthetic valve with respect to the native annulus and the coronary ostia. The average procedure duration from skin incision to closure including scanning was less than 40 minutes.

Retrospectively gated cine-MRI revealed excellent myocardial function after valve implantation in both long- and short-axis views (snapshot is shown in Figure 3, column 1, row 1 and row 2). The phase-contrast cine-MRIs (Figure 3, column 1, row 3) show the through-plane blood velocity in both systole and diastole. These images confirm good systolic flow with excellent valve leaflet opening and no evidence of turbulence, diastolic regurgitant flow, or paravalvular leak. First-pass perfusion studies demonstrated adequacy of myocardial blood flow after valve placement in all animals after successful deployment. A first-pass perfusion scan at the base of the heart after valve implantation is shown in Figure 3, column 1, row 4. These perfusion results confirm adequacy of blood flow at the tissue level, indicating proper valve positioning with respect to the coronary ostia. Echocardiographic results confirmed the MRI findings and further documented the stability of prosthesis position and function over time. Necropsy of all these animals confirmed the deployed prosthetic valve location with respect to the aortic annulus and the mitral valve, and the commissures did not obstruct the coronary ostia. The observed distances between the commissural marker and a coronary ostium were $4 \mathrm{~mm}$ or greater. These necropsy findings verified the MRI and echocardiography results.

Among 10 animals allowed to survive, 7 survived for 6 months. Three animals died between 1 and 2 weeks postoperatively of respiratory complications. The 7 long-term survivors were brought back for echocardiography and MRI scan 1,3 , and 6 months after the procedure. Figure 3 shows long-term follow-up MRI scan for a pig at 1, 3, and 6 months, with regard to anatomic positioning, phase contrast, and perfusion. In the follow-up studies, perfusion scanning demonstrated adequate coronary flow and functional imaging documented preservation of ventricular contractility.
Aortic Valve Regurgitation

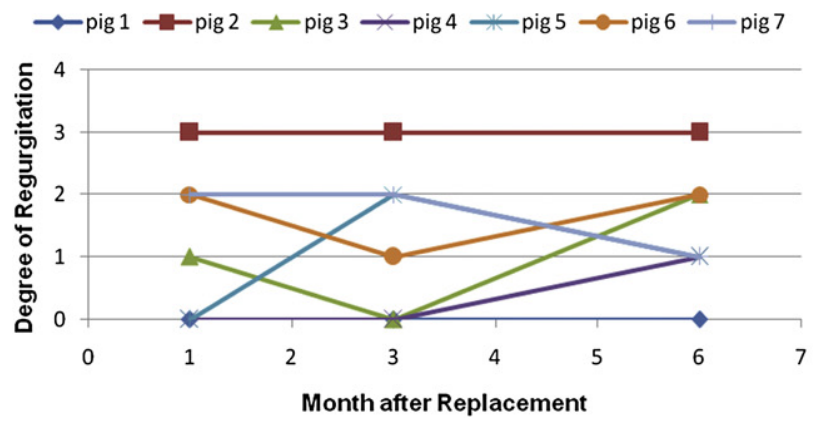

\section{Mitral Valve Regurgitation}

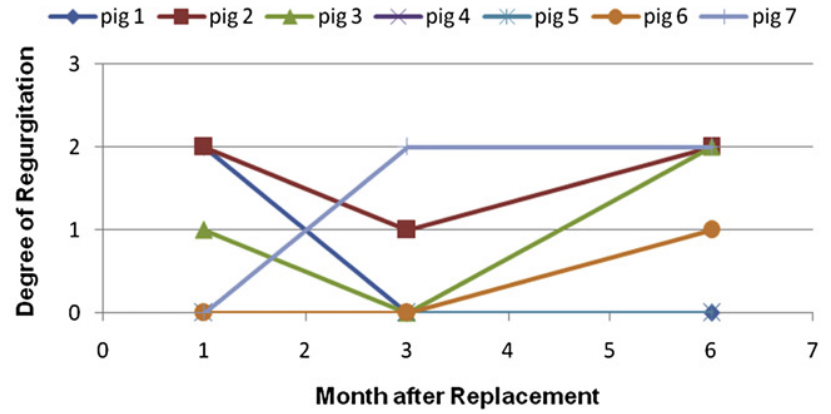

FIGURE 4. Aortic valve and mitral valve regurgitation of 7 animals with long-term survival. 
Phase contrast imaging revealed minimal intravalvular or paravalvular leaks. The ventricular and valvular parameters of 7 animals based on 1,3, and 6 months' follow-up echocardiograms and MRI scan are presented in Table 1. Analysis of variance was performed to determine the significance of the differences between the 1-, 3-, and 6-month data. The $P$ values are also shown in Table 1. From these $P$ values we can conclude that there is no significant difference among the ventricular and valvular parameters during the 6 months after the procedure. The degree of insufficiency of the aortic valve and the mitral valve over 6 months after the procedure were measured and shown in Figure 4. Longer term results demonstrated stability of the implants without migration or change in position and with preservation of myocardial perfusion and function over time. The histopathologic reports of midterm (6 months) verified that all the implanted aortic stent and valve assemblies were in place in the aortic root. The stents were properly seated in firm apposition to the aortic wall from the level of the prosthetic leaflets to the distal commissures tips. The prosthetic device leaflets remained cusp shaped and in the closed position exhibited good coaptation of the free edges. These necropsy findings correlated with both the MRI and echocardiographic results. Two animals grew from $50 \mathrm{~kg}$ to $200 \mathrm{~kg}$ during the 6-month period of follow-up; and the aortas partially outgrew the original implanted aortic stents. At necropsy after 6 months in these animals, some focal gaps were observed between the device and the native aortic wall in the area of the native aortic leaflet. These gaps allowed for perivalvular regurgitation, which was noted on MRI and echocardiogram before necropsy.

\section{DISCUSSION}

Percutaneous transfemoral aortic valve replacement has been reported in a highly selected cohort of patients. ${ }^{2}$ The percutaneous technique requires excessive minimization of the prosthesis, which still remains bulky, could result in device embolization, and is difficult to traverse undersized, calcified, or tortuous iliofemoral vessels. ${ }^{15}$ Transapical aortic valve implantation is a relatively easy, safe, and straightforward direct technique, which allows excellent alignment between the prosthesis and the aortic root. Patients with symptomatic, severe aortic stenosis and high surgical risk are more suitable for the transapical approach. ${ }^{16,17}$

According to the literature, in both the percutaneous transfemoral and transapical approaches, positioning of the prosthesis is mostly aided by 2-dimensional M-mode transesophageal echocardiography and high-resolution fluoroscopy. ${ }^{7}$ Fluoroscopy provides little soft tissue contrast. Additionally, what weakly visible landmarks that the calcification of the stenotic valve provides are lost or distorted after the mandatory balloon valvuloplasty done before valve placement. Contrast agents must be frequently injected to determine the location of the aortic annulus and coronary ostia. The patient and physician are exposed to radiation during the intervention. Echocardiography is dependent on ultrasonic beam reflection and therefore is subject to impaired imaging caused by limited acoustic windows. This is particularly true for the aortic valve when considering the impact of calcification and/or prosthesis shadowing. Fusion imaging combining computed tomography and fluoroscopy has been proposed to overcome these issues but are not real time, are highly dependent on stable patient registration, and significantly increase the radiation exposure. Clinical results of both transfemoral and transapical approaches for percutaneous aortic valve replacement have reported success with these techniques. Thus far, these procedures have carried a significant morbidity and mortality. Major adverse cardiac and cerebrovascular events have been seen in $32 \%$ to $65 \%$ of the patients. ${ }^{15}$ Periprocedural mortality has ranged from $10 \%$ to $23 \% .{ }^{18,19}$ Although some of these outcomes are due to the comorbidities of the high-risk patients being treated, many can be attributed to imaging-related problems including coronary obstruction, myocardial infarction, device misalignment, and valve embolization. In fact, valve malposition or migration are two of the top three reasons for procedural failure. ${ }^{20}$ Other frequently reported limitations that are imaging related include landmark loss after balloon valvuloplasty, perivalvular leak owing to inability to determine whether the stent is adequately opposed to the aorta and aortic annulus, and fatal arrhythmias resulting from the rapid ventricular pacing required to provide time for valve placement. These difficulties should be decreased by improving the imaging used to perform the procedure.

Real-time MRI provides excellent visualization for intraoperative guidance of aortic valve replacement on the beating heart. It provides better image quality and a complete view of the entire chest more than other competing imaging methods, such as fluoroscopy/angiography, in which some anatomic structures are not visible, and echocardiography, in which the field of view is small. MRI-guided surgery also allows direct functional assessments to be made before, during, and immediately after valve implantation that are not obtainable by conventional imaging alone. ${ }^{21}$

Expansion of real-time MRI guidance to facilitate other types of cardiac surgical procedures, including mitral, pulmonary, and tricuspid valve replacements or repairs, should be considered to minimize trauma and enhance patient benefit.

\section{References}

1. Boudjemline Y, Bonhoeffer P. Steps toward percutaneous aortic valve replacement. Circulation. 2000;105:775-8.

2. Cribier A, Eltchaninoff H, Tron C, Bauer F, Agatiello C, Nercolini D, et al. Treatment of calcific aortic stenosis with the percutaneous heart valve: mid-term follow-up from the initial feasibility studies: The French experience. J Am Coll Cardiol. 2006;47:1214-23.

3. Grube E, Laborde JC, Zickmann B, Gerckens U, Felderhoff T, Sauren B, et al First report on a human percutaneous transluminal implantation of a self-expanding valve prosthesis for interventional treatment of aortic valve stenosis. Catheter Cardiovasc Interv. 2005;66:465-9. 
4. Huber C, Cohn LH, von Segesser LK. Direct-access valve replacement: a novel approach for off-pump valve implantation using valved stents. J Am Coll Cardiol. 2005;46:366-70.

5. Lichtenstein SV, Cheung A, Ye J, Thompson CR, Carere RG, Pasupati S, et al Transapical transcatheter aortic valve implantation in humans: initial clinical experience. Circulation. 2006;114:591-6.

6. Walther T, Falk V, Kempfert J, Borger MA, Fassl J, Chu MW, et al. Transapical minimally invasive aortic valve implantation; the initial 50 patients. Eur J Cardiothorac Surg. 2008;33:983-8.

7. Walther T, Dewey T, Borger MA, Kempfert J, Linke A, Becht R, et al. Transapical aortic valve implantation: step by step. Ann Thorac Surg. 2009;87: 276-83.

8. Webb JG, Pasupati S, Humphries K, Thompson C, Altwegg L, Moss R, et al. Percutaneous transarterial aortic valve replacement in selected high-risk patients with aortic stenosis. Circulation. 2007;116:755-63.

9. Ye J, Cheung A, Lichtenstein SV, Pasupati S, Carere RG, Thompson CR, et al. Six-month outcome of transapical transcatheter aortic valve implantation in the initial seven patients. Eur J Cardiothorac Surg. 2007;31:16-21.

10. McVeigh ER, Guttman MA, Lederman RJ, Li M, Kocaturk O, Hunt T, et al. Realtime interactive MRI-guided cardiac surgery: aortic valve replacement using a direct apical approach. Magn Reson Med. 2006;56:958-64.

11. Horvath KA, Guttman M, Li M, Lederman RJ, Mazilu D, Kocaturk O, et al. Beating heart aortic valve replacement using real-time MRI guidance. Innovation. 2007;2:51-5.

12. Guttman MA, Kellman P, Dick AJ, Lederman RJ, McVeigh ER. Real-time accelerated interactive MRI with adaptive TSENSE and UNFOLD. Magn Reson Med. 2003;50:315-21.
13. Lederman RJ. Cardiovascular interventional magnetic resonance imaging. Circulation. 2005;112:3009-17.

14. Kellman P, Derbyshire JA, Agyeman KO, McVeigh ER, Arai AE. Extended coverage first-pass perfusion imaging using slice-interleaved TSENSE. Magn Reson Med. 2004;51:200-4.

15. Zajarias A, Cribier AG. Outcomes and safety of percutaneous aortic valve replacement. J Am Coll Cardiol. 2009;53:1829-36.

16. Bonow RO, Carabello BA, Chatterjee K, de Leon AC, Faxon DP, Freed MD, et al. ACC/AHA 2006 Guidelines for the management of patients with valvular heart disease: executive summary. Circulation. 2006;114:450-527.

17. Vahanian A, Alfieri O, Al-Attar N, Antunes M, Bax J, Cormier B, et al. Transcatheter valve implantation for patients with aortic stenosis: a position statement from the European Association of Cardio-Thoracic Surgery (EACTS) and the European Society of Cardiology (ESC), in collaboration with the European Association of Percutaneous Cardiovascular Interventions (EAPCI). Eur Heart J. 2008;29:1463-70.

18. Laborde JC. Transcatheter aortic valve implantation with the CoreValve ReValving Device. Presented at Transcatheter Cardiovascular Therapeutics. 2008 Oct 12; Washington DC.

19. Cribier A, Eltchaninoff H, Tron C, Bauer F, Agatiello C, Sebagh L, et al. Early experience with percutaneous transcatheter implantation of heart valve prosthesis for the treatment of end stage inoperable patients with calcific aortic stenosis. J Am Coll Cardiol. 2004;43:698-703.

20. Walther T. Transapical aortic valve implantation: Traverse Feasibility Study. Presented at Transcatheter Cardiovascular Therapeutics; 2008 Oct 13; Washington DC.

21. Hasenkam JM, Ringgaard S, Houlind K, Botnar RM, Stodkilde-Jorgensen H, Boesiger $\mathrm{P}$, et al. Prosthetic heart valve evaluation by magnetic resonance imaging. Eur J Cardiothorac Surg. 1999;16:300-5. 